\title{
Non-matching Grids and Lagrange Multipliers
}

\author{
S. Bertoluzza ${ }^{1}$, F. Brezzi ${ }^{1,2}$, L.D. Marini ${ }^{1,2}$, and G. Sangalli ${ }^{1}$ \\ 1 Istituto di Matematica Applicata e Tecnologie Informatiche del C.N.R., Pavia

 \\ 2 Università di Pavia, Dipartimento di Matematica
}

Summary. In this paper we introduce a variant of the three-field formulation where we use only two sets of variables. Considering, to fix the ideas, the homogeneous Dirichlet problem for $-\Delta u=g$ in $\Omega$, our variables are $i$ ) an approximation $\psi_{h}$ of $u$ on the skeleton (the union of the interfaces of the sub-domains) on an independent grid (that could often be uniform), and ii) the approximations $u_{h}^{s}$ of $u$ in each subdomain $\Omega^{s}$ (each on its own grid). The novelty is in the way to derive, from $\psi_{h}$, the values of each trace of $u_{h}^{s}$ on the boundary of each $\Omega^{s}$. We do it by solving an auxiliary problem on each $\partial \Omega^{s}$ that resembles the mortar method but is more flexible. Optimal error estimates are proved under suitable assumptions.

\section{Introduction}

Assume, for simplicity, that we have to solve the model problem

$$
\text { find } u \in H_{0}^{1}(\Omega) \text { such that } \quad-\Delta u=g \text { in } \Omega \quad \text { with } u=0 \text { on } \partial \Omega
$$

on a polygonal or polyhedral domain $\Omega \subset \mathbb{R}^{n}, n=2,3$, where $g$ is a given function sufficiently regular in $\Omega$. In order to apply a Domain Decomposition technique we split $\Omega$ into sub-domains $\Omega^{s}(s=1,2, \ldots, S)$ and we consider the skeleton

$$
\Sigma:=\cup_{s} \Gamma^{s}, \quad \text { with } \quad \Gamma^{s} \equiv \partial \Omega^{s} .
$$

For the sake of simplicity we will use a three-dimensional notation, and speak therefore of faces, edges and vertices. The change of terminology in the polygonal case is obvious and left to the reader. On $\Sigma$ we consider

$$
\Phi:=\left\{\varphi \in L^{2}(\Sigma): \exists v \in H_{0}^{1}(\Omega) \text { with } \varphi=v_{\mid \Sigma}\right\} \equiv H_{0}^{1}(\Omega)_{\mid \Sigma} \equiv H^{1 / 2}(\Sigma) .
$$

In each $\Omega^{s}$ we consider instead

$$
V^{s}:=\left\{v^{s} \in H^{1}\left(\Omega^{s}\right) \text { such that } \exists v \in H_{0}^{1}(\Omega) \text { with } v^{s}=v_{\mid \Omega^{s}}\right\},
$$


that can also be seen as the set of functions in $H^{1}\left(\Omega^{s}\right)$ that vanish at the intersection (if any) of $\Gamma^{s}$ with $\partial \Omega$. In its turn, $H_{0}^{1}(\Omega)$ could be identified with a subspace of

$$
V:=\left\{u \in L^{2}(\Omega),\left.u\right|_{\Omega^{s}} \in V^{s}\right\},
$$

and in particular, setting $v^{s}:=\left.v\right|_{\Omega^{s}}$ we can write

$$
H_{0}^{1}(\Omega) \simeq\left\{v \in V \text { such that } \exists \varphi \in \Phi \text { with } v^{s}=\varphi \text { on } \Gamma^{s}, \quad s=1, \ldots, S\right\} .
$$

For each $s$ we will also introduce the trace space $\Theta^{s}=H^{1 / 2}\left(\Gamma^{s}\right)$, and we set $\Theta=\prod_{s} \Theta^{s}$. For $v \in V, \theta=\left(\theta^{1}, \ldots, \theta^{s}\right) \in \Theta$ we will write

$$
\left.v\right|_{\Sigma}=\theta \text { to indicate that } \theta^{s}=\left.v^{s}\right|_{\Gamma^{s}}\left(\text { with } v^{s}=\left.v\right|_{\Omega^{s}}\right), \quad s=1, \ldots, S .
$$

When discretizing the problem, we assume to be given a decomposition $\mathcal{T}_{\delta}^{\Sigma}$ of $\Sigma$ and a corresponding space $\Phi_{\delta} \subset \Phi$ of piecewise polynomials. We also assume that in each $\Omega^{s}$ we are given a decomposition $\mathcal{T}_{h}^{s} \equiv \mathcal{T}_{h}^{\Omega^{s}}$ with a corresponding space $V_{h}^{s} \subset V^{s}$ of piecewise polynomials, and we set

$$
V_{h}:=\left\{v \in V \text { such that }\left.v\right|_{\Omega^{s}} \in V_{h}^{s}\right\} .
$$

It is clear that each decomposition $\mathcal{T}_{h}^{s}$ will induce a decomposition $\mathcal{T}_{h}^{\Gamma^{s}}$ on $\Gamma^{s}$ and a corresponding space of traces $\Theta_{h}^{s} \subset \Theta^{s}$. On the other hand the restriction of $\mathcal{T}_{\delta}^{\Sigma}$ to $\Gamma^{s}$ also induces a decomposition $\mathcal{T}_{\delta}^{\Gamma^{s}}$ of $\Gamma^{s}$ and another space of piecewise polynomials $\Phi_{\delta}^{s}$ made by the restrictions of the functions in $\Phi_{\delta}$ to $\Gamma^{s}$. Hence, on each $\Gamma^{s}$ we have two decompositions (one coming from $\mathcal{T}_{\delta}^{\Sigma}$ and one from $\mathcal{T}_{h}^{s}$ ) and two spaces of piecewise polynomial functions (one from $\Phi_{\delta}$ and one from $V_{h}^{s}$ ). Note, incidentally, that on each face $f$ belonging to two different sub-domains we will have three decompositions and three spaces: one from $\Sigma$ and the other two from the two sub-domains.

The first basic idea of our method is to design for every sub-domain $\Omega^{s}$ a linear operator $\mathcal{G}^{s}$ (the generation operator) that maps every mother $\varphi_{\delta} \in \Phi_{\delta}$ into an element (daughter) $\theta_{h}^{s}=\mathcal{G}^{s}\left(\varphi_{\delta}\right) \in \Theta_{h}^{s}$. Together with the individual $\mathcal{G}^{s}$ we consider a global operator $\mathcal{G}$ defined as

$$
\mathcal{G}\left(\varphi_{\delta}\right)=\left(\theta_{h}^{1}, \ldots, \theta_{h}^{S}\right) \in \Theta_{h} \quad \text { with } \theta^{s}=\mathcal{G}^{s}\left(\varphi_{\delta}\right) .
$$

The way to construct the operators $\mathcal{G}^{s}$ constitutes the second basic idea of this paper, and will be described in a while.

Once we have the operators $\mathcal{G}^{s}$ we can consider the subspace $\mathcal{S}_{h}$ of $V_{h}$ made of sisters (that is, daughters of the same mother):

$$
\mathcal{S}_{h}:=\left\{v_{h} \in V_{h} \text { such that } \exists \varphi_{\delta} \in \Phi_{\delta} \text { with } v_{h \mid \Sigma}=\mathcal{G}\left(\varphi_{\delta}\right)\right\} \subseteq V .
$$

We point out that in our previous definitions we consider as daughter, at the same time, an element $\theta_{h}^{s}\left(=\left.v_{h}^{s}\right|_{\Gamma^{s}}\right)$ of $\Theta_{h}^{s}$, and any function $v_{h}^{s} \in V_{h}^{s}$ having that same trace. It is clear, comparing (9) with (6), that $\mathcal{S}_{h}$ can be seen 
as a nonconforming approximation of $H_{0}^{1}(\Omega)$. This allows us to consider the following discrete formulation. We set

$$
a_{s}(u, v):=\int_{\Omega^{s}} \nabla u \cdot \nabla v \mathrm{~d} x \quad \text { and } \quad a(u, v):=\sum_{s=1}^{S} a_{s}\left(u^{s}, v^{s}\right)
$$

and we look for $u_{h} \in \mathcal{S}_{h}$ such that

$$
a\left(u_{h}, v_{h}\right)=\int_{\Omega} g v_{h} \mathrm{~d} x \quad \forall v_{h} \in \mathcal{S}_{h} .
$$

It is clear that, under reasonable assumptions on the subspaces $\Phi_{\delta}$ and $V_{h}^{s}$ and on the generation operators $\mathcal{G}^{s}$, problem (11) will have good stability and accuracy properties.

The idea of imposing weak continuity by introducing the space $\Phi_{\delta}$ and define a nonconforming approximation of $H_{0}^{1}(\Omega)$ by taking the subset of $V_{h}$ whose elements take (in some weak sense) value $\varphi_{h} \in \Phi_{\delta}$ is one of the main ideas of the three field formulation (Brezzi and Marini [1994]). Following that approach, for each sub-domain $\Omega^{s}$ we could take a space $M_{h}^{s}$ of Lagrange multipliers, and, for every $\varphi_{\delta} \in \Phi_{\delta}$, we could define $\mathcal{G}^{s}\left(\varphi_{\delta}\right) \in \Theta_{h}^{s}$ by

$$
\int_{\Gamma^{s}}\left(\varphi_{\delta}-\mathcal{G}^{s}\left(\varphi_{\delta}\right)\right) \mu_{h}^{s} \mathrm{~d} x=0 \quad \forall \mu_{h}^{s} \in M_{h}^{s} .
$$

In general, however, equation (12) does not define $\mathcal{G}^{s}\left(\varphi_{d}\right.$ elta) uniquely, even when the spaces $M_{h}^{s}$ and $\Theta_{h}^{s}$ satisfy the required inf-sup condition (see (24)). Though this is not a problem in the definition and in the analysis of the three field formulation, we would like to point out that having the trace of the elements $v_{h}^{s}$ on $\Gamma^{s}$ somehow uniquely determined by an element of $\Phi_{\delta}$ has some clear advantage from the point of view of implementation. In particular it allows to use standard Dirichlet solvers (which can easily be found already implemented and whose optimization is well understood) as a brick for treating the equation in the subdomain. In order for $\mathcal{G}^{s}\left(\varphi_{\delta}\right)$ to be uniquely determined by (12) the spaces $M_{h}^{s}$ and $\Theta_{h}^{s}$ must have the same dimension. A simple minded choice is $M_{h}^{s} \equiv \Theta_{h}^{s}$, that guarantees existence and uniqueness of the solution of (12) together with optimal stability and accuracy properties of the projector $\mathcal{G}^{s}$. This choice however is not the optimal one: in fact, during the estimate of the error for problem (11), there seems to be no way to get rid of a term like

$$
\sum_{s} \int_{\Gamma^{s}} \frac{\partial u}{\partial \mathbf{n}_{s}}\left(\varphi_{\delta}-\mathcal{G}^{s}\left(\varphi_{\delta}\right)\right) \mathrm{d} x
$$

An obvious way to treat the term in (13) is to use the fact that $\varphi_{\delta}-\mathcal{G}^{s}\left(\varphi_{\delta}\right)$ is orthogonal to all elements in $M_{h}^{s}$, so that we can subtract from $\partial u / \partial \mathbf{n}_{s}$ any element of $M_{h}^{s}$. In particular we are interested in subtracting a suitable approximation $\mu_{I}^{s} \simeq \partial u / \partial \mathbf{n}_{s}$. It is then crucial to be able to find in $M_{h}^{s}$ a $\mu_{I}^{s}$ that approximates $\partial u / \partial \mathbf{n}_{s}$ with the needed order. However, $\partial u / \partial \mathbf{n}_{s}$ is 
discontinuous passing from one face to another of the same $\Omega^{s}$. And if the space $M_{h}^{s}$ is made of continuous functions (as it would be with the choice $M_{h}^{s} \equiv$ $\Theta_{h}^{s}$ ), then the order of approximation (say, in $H^{-1 / 2}\left(\partial \Omega^{s}\right)$ ) cannot be better than $O(h)$ (and actually with some additional logarithmic loss, as $O(h|\lg h|)$. Hence, we do need an $M_{h}^{s}$ made of functions that can be discontinuous when passing from one face to another of the same $\Omega^{s}$. The requirement to contain a suitable amount of discontinuities and the one to have the same dimension of $\Theta_{h}^{s}$ seem very difficult to conciliate. Actually, a quite similar difficulty is met in the mortar method, (see e.g. Bernardi et al. [1993], Belgacem and Maday [1997], Hoppe et al. [1998], Wohlmuth [2001]), in particular in three dimensions. There, the requirement that $M_{h}^{s}$ have the same dimension as $\Theta_{h}^{s}$ is relaxed as little as possible. The values of a "weakly continuous" function $v_{h}^{s}$ at nodes which are interior to the faces of $\Gamma^{s}$ on the slave sides are uniquely determined by the weak continuity equation, while the degrees of freedom corresponding to nodes on the edges of $\Gamma^{s}$ (whose union forms the so called wirebasket) are free. We point out that the mortar method can be described in the framework given here provided we relax the assumption $\Phi_{\delta} \subset H^{1 / 2}(\Sigma)$ by allowing the functions $\phi_{\delta}$ to be discontinuous across the "wirebasket": $\Phi_{\delta}$ would correspond to the traces of $v_{h}$ on the "master sides" (or "mortars") and $\mathcal{G}^{s}$ being defined as the identity on master sides and to one of the available mortar projections on "slave sides".

The idea, here, is to give up the equality of the dimensions but still obtain a well defined operator $\mathcal{G}^{s}$, by changing (12) in a slightly more complicated formulation, involving an additional Lagrange multiplier. Let us see the main features of this path.

We choose first a space $M_{h}^{s}$ having in mind the fact that we must be able to use it for approximating $\partial u / \partial \mathbf{n}_{s}$ with the right order. We also need its dimension to be smaller than (or equal to) that of $\Theta_{h}^{s}$. Then we change (12) in the following way. For every $\varphi_{\delta} \in \Phi_{\delta}$ we look for a pair $\left(\widetilde{\theta_{h}^{s}}, \widetilde{\mu_{h}^{s}}\right)$ in $\Theta_{h}^{s} \times M_{h}^{s}$ such that

$$
\int_{\Gamma^{s}}\left(\varphi_{\delta}-\widetilde{\theta_{h}^{s}}\right) \mu_{h}^{s} \mathrm{~d} x=0 \quad \forall \mu_{h}^{s} \in M_{h}^{s}
$$

and

$$
\sum_{T \in \mathcal{T}_{h}^{\Gamma^{s}}} \int_{T} h_{T}^{-1}\left(\varphi_{\delta}-\widetilde{\theta_{h}^{s}}\right) \theta_{h}^{s} \mathrm{~d} x+\int_{\Gamma^{s}} \widetilde{\mu_{h}^{s}} \theta_{h}^{s} \mathrm{~d} x=0 \quad \forall \theta_{h}^{s} \in \Theta_{h}^{s} .
$$

Then we set

$$
\mathcal{G}^{s}\left(\varphi_{\delta}\right):=\widetilde{\theta_{h}^{s}}
$$

It is clear that in (14)-(15) the number of equations will always be equal to the number of unknowns. It is also clear that if (by shear luck) we have $\left.\varphi_{\delta}\right|_{\Gamma^{s}} \in \Theta_{h}^{s}$ then $\mathcal{G}^{s}\left(\varphi_{\delta}\right)=\left.\varphi_{\delta}\right|_{\Gamma^{s}}$ (and $\widetilde{\mu_{h}^{s}}=0$ ). This will, in the end, provide for the new approach (14)-(16) an optimal order of accuracy (as we had for the previous simple-minded (12)). It is, finally, also obvious that some sort of 
inf-sup condition will be needed in order to ensure existence and uniqueness of the solution of (14)-(15), unless some suitable additional stabilization is introduced. However, as we shall see, the possibility of escaping the cage of the equal dimensionality of $M_{h}^{s}$ and $\Theta_{h}^{s}$ opens a whole lot of interesting possibilities.

In this paper we will follow the path indicated above. In the next section we will make precise all the necessary assumptions, and in Section 3 we will derive abstract error bounds for problem (11) when the operators $\mathcal{G}^{s}$ are constructed as in (14)-(16). In Section 4 we will present some possible choices for the finite element spaces and discuss their stability and accuracy properties. In particular we will show that the simple choice of using totally discontinuous functions for $M_{h}^{s}$, stabilizing the problem with suitable boundary bubbles, leads to a problem with optimal convergence properties and, at the same time, a very simple implementation. This is reminiscent of what has been done for instance in Baiocchi et al. [1992], Brezzi et al. [1997], Buffa [2002], and Brezzi and Marini [2000], but simpler and more effective. Finally, in the last section we briefly discuss some possible variants/extensions, in particular regarding the possibility of using discontinuous mothers.

\section{Assumptions on the decomposition and on the discretizations}

We consider now the assumptions to be made on the decomposition and on the discretizations.

\section{Assumptions on $\Omega$ and on the domain decomposition}

We assume that $\Omega$ is an open polyhedron, that each $\Omega^{s}$, for $s=1, \ldots, S$, is also an open polyhedron, that the intersection of two different $\Omega^{s}$ is empty, and that the union of the closures of all $\Omega^{s}$ is the closure of $\Omega$. As in (2) the skeleton $\Sigma$ will be the union of the boundaries $\partial \Omega^{s}$. We do not assume that this decomposition is compatible. This means that we do not assume that the intersection of the closure of two different $\Omega^{s}$ is either a common face, or a common edge, or a common vertex. For simplicity we assume however that the number $S$ of subdomains is fixed once and for all, and we do not keep track of the dependency of the various constants on $S$.

\section{Assumptions on the decomposition $\mathcal{T}_{\delta}^{\Sigma}$}

We assume that we are given a sequence $\left\{\mathcal{T}_{\delta}^{\Sigma}\right\}_{\delta}$ of decompositions of $\Sigma$. Each decomposition $\mathcal{T}_{\delta}^{\Sigma}$ is made of open triangles, in such a way that the intersection of two different triangles is empty, and the union of the closures of all triangles is $\Sigma$. We assume compatibility, that is we assume that the 
intersection of the closures of two different triangles is either empty, a common edge or a common vertex. We also assume, as usual, shape regularity, for instance by assuming that the ratio between the diameter of each triangle and the radius of its biggest inscribed circle is $\leq \kappa_{0}$, with $\kappa_{0}$ independent of $\delta$. Finally we assume quasi-uniformity: there exists a constant $q$, independent of $\delta$ such that, if $\delta_{T}^{\min }$ and $\delta_{T}^{\max }$ are the minimum and the maximum diameters (respectively) of the triangles in $\mathcal{T}_{\delta}^{\Sigma}$, then $\delta_{T}^{\text {min }} \geq q \delta_{T}^{\max }$.

\section{Assumptions on the decompositions $\mathcal{T}_{h}^{s}\left(\right.$ and $\left.\mathcal{T}_{h}^{\Gamma^{s}}\right)$}

We assume that we are given, for each $s=1, \ldots, S$, a sequence $\left\{\mathcal{T}_{h}^{s}\right\}_{h}$ of decompositions of $\Omega^{s}$. Each decomposition is made of open tetrahedra in such a way that the intersection of two different tetrahedra is empty, and the union of the closures of all tetrahedra is $\Omega^{s}$. We also assume compatibility: the intersection of the closures of two different tetrahedra is either empty, a common face, a common edge, or a common vertex. Finally we assume shape regularity, for instance by assuming that the ratio between the diameter of each tetrahedron and the radius of its biggest inscribed sphere is $\leq \kappa_{1}$, with $\kappa_{1}$ independent of $h$. We point out that we do not assume quasi-uniformity for the meshes $\mathcal{T}_{h}^{s}$. We recall that the triangulation $\mathcal{T}_{h}^{\Gamma^{s}}$ is the restriction on $\Gamma^{s}$ of $\mathcal{T}_{h}^{s}$.

\section{Assumptions on the discretizations $\Phi_{\delta}, V_{h}^{s}$, and $M_{h}^{s}$}

We assume that for each $\delta$ and for each $T \in \mathcal{T}_{\delta}^{\Sigma}$ we are given a space of polynomials $\mathcal{P}_{T}$. The space $\Phi_{\delta}$ will then be defined as

$$
\Phi_{\delta}:=\left\{\varphi \in \Phi \text { such that } \varphi_{\mid T} \in \mathcal{P}_{T}, \quad T \in \mathcal{T}_{\delta}^{\Sigma}\right\},
$$

where $\Phi$ is always given by (3). Similarly we assume that for each $s$, for each $h$, and for each $K \in \mathcal{T}_{h}^{s}$ we are given a space of polynomials $\mathcal{P}_{K}$. The space $V_{h}^{s}$ will then be defined as

$$
V_{h}^{s}:=\left\{v^{s} \in V^{s} \text { such that } v_{\mid K}^{s} \in \mathcal{P}_{K}, \quad K \in \mathcal{T}_{h}^{s}\right\},
$$

where $V^{s}$ is still given by (4).

The corresponding restrictions of the above spaces to each $\Gamma^{s}$ are defined as in the previous section, namely

$$
\Phi_{\delta}^{s}:=\left(\Phi_{\delta}\right)_{\mid \Gamma^{s}} \quad \text { and } \quad \Theta_{h}^{s}:=\left(V_{h}^{s}\right)_{\mid \Gamma^{s}}, \quad s=1, \ldots, S .
$$

We assume that there exist bounded lifting operators from $\Theta_{h}^{s}$ to $V_{h}^{s}$. More precisely, for all $s=1, \ldots, S$, for all $\theta_{h}^{s} \in \Theta_{h}^{s}$ there exists $w_{h}^{s} \in V_{h}^{s}$ such that

$$
\left.w_{h}^{s}\right|_{\Gamma^{s}}=\theta_{h}^{s} \quad \text { and } \quad\left\|w_{h}^{s}\right\|_{1, \Omega^{s}} \leq C\left\|\theta_{h}^{s}\right\|_{H^{1 / 2}\left(\Gamma^{s}\right)} .
$$


Finally we assume that for each $s$, for each $h$, and for each $T \in \mathcal{T}_{h}^{\Gamma^{s}}$ we are given a space of polynomials $\mathcal{Q}_{T}$. The space $M_{h}^{s}$ will then be defined as

$$
M_{h}^{s}:=\left\{\mu \in L^{2}\left(\Gamma^{s}\right) \text { such that } \mu_{\mid T} \in \mathcal{Q}_{T}, \quad T \in \mathcal{T}_{h}^{\Gamma^{s}}\right\} .
$$

If we like, we can also add some continuity requirements to (21). In view of the discussion of the previous section, however, it would be unwise to force continuity in the passage from one face to another. In order for the bilinear form $a$ to ve coercive in a suitable space, we make the following minimal assumption on $M_{h}^{s}$ :

$$
\text { for every } \Omega^{s} \text { the space } M_{h}^{s} \text { contains the constants on } \Gamma^{s} \text {. }
$$

Moreover, for simplicity, we assume that there exists an integer number $\kappa$ such that all the spaces $\mathcal{P}_{T}, \mathcal{P}_{K}$, and $\mathcal{Q}_{T}$ verify

$$
\mathcal{P}_{T} \subseteq \mathbb{P}_{\kappa}(T), \quad \mathcal{P}_{K} \subseteq \mathbb{P}_{\kappa}(K), \quad \mathcal{Q}_{T} \subseteq \mathbb{P}_{\kappa}(T),
$$

where $\mathbb{P}_{\kappa}(\omega)$ is the space of polynomials of degree $\leq \kappa$ on $\omega$. Using the notation of Brezzi and Fortin [1991a] for the usual Lagrange finite element spaces we can then write

$$
V_{h}^{s} \subseteq \mathcal{L}_{\kappa}^{1}\left(\mathcal{T}_{h}^{s}\right), \quad \Theta_{h}^{s} \subseteq \mathcal{L}_{\kappa}^{1}\left(\mathcal{T}_{h}^{\Gamma^{s}}\right), \quad M_{h}^{s} \subseteq \mathcal{L}_{\kappa}^{0}\left(\mathcal{T}_{h}^{\Gamma^{s}}\right), \quad \Phi_{\delta} \subseteq \mathcal{L}_{\kappa}^{1}\left(\mathcal{T}_{\delta}^{\Sigma}\right) .
$$

\section{The operators $\mathcal{G}^{s}$ and the compatibility assumptions among the discretizations}

Having defined the spaces $\Theta_{h}^{s}$ and $M_{h}^{s}$ we can now consider the operators $\mathcal{G}^{s}$ (that will always be given by (14)-(16)) together with the global operator $\mathcal{G}$ (still given by $(8)$ ). Once we have the operators $\mathcal{G}^{s}$ and $\mathcal{G}$, we can define the space of sisters $\mathcal{S}_{h}$, always as in (9). In $\mathcal{S}_{h}$ we define:

$$
\left\|v_{h}\right\|^{2}:=\sum_{s=1}^{S}\left\|\nabla v_{h}^{s}\right\|_{0, \Omega^{s}}^{2}
$$

We can now turn to the more important assumptions, that will require some compatibility conditions among the spaces $\Phi_{\delta}^{s}, \Theta_{h}^{s}$ and $M_{h}^{s}$.

Our first assumption will deal with the well-posedness of the problem (14)(16). As this is a problem in classical mixed form, we have no real escape but assuming an inf-sup condition on the spaces $\Theta_{h}^{s}$ and $M_{h}^{s}$ :

$\exists \beta>0$ such that $\forall s=1, \ldots, S$ and $\forall h>0$

$$
\inf _{\mu_{h}^{s} \in M_{h}^{s} \backslash\{0\}} \sup _{\theta_{h}^{s} \in \Theta_{h}^{s} \backslash\{0\}} \frac{\int_{\Gamma^{s}} \theta_{h}^{s} \mu_{h}^{s} \mathrm{~d} x}{\left\|\theta_{h}^{s}\right\|_{h, \frac{1}{2}, \Gamma^{s}}\left\|\mu_{h}^{s}\right\|_{h,-\frac{1}{2}, \Gamma^{s}}}>\beta,
$$

were the norms in the denominator of (24) are defined, for any real $r$, as 


$$
\left\|\theta_{h}^{s}\right\|_{h, r, \Gamma^{s}}^{2}:=\sum_{T \in \mathcal{T}_{h}^{\Gamma^{s}}} h_{T}^{-2 r}\left\|\theta_{h}^{s}\right\|_{0, T}^{2}
$$

and $h_{T}$ is the diameter of $T$. Condition (24) will be, in a sense, the only nontrivial assumption that we have to take into account in the definition of our spaces $V_{h}^{s}$ and $M_{h}^{s}$. However, in the next section, we are going to see some families of elements where (24) can be checked rather easily.

Our last assumption will deal with the bound on the mother. We point out that, so far, we did not assume that an element of the space of sisters $\mathcal{S}_{h}$ had a unique mother. Indeed, we do not need it. Strictly speaking, we only need that

$$
\begin{gathered}
\exists \gamma>0 \text { such that: } \forall v_{h} \in \mathcal{S}_{h}, \exists \varphi_{\delta} \in \Phi_{\delta} \text { with } \mathcal{G}\left(\varphi_{\delta}\right)=v_{h \mid \Sigma} \text { and } \\
\|\varphi\|_{\Phi}^{2}:=\sum_{s=1}^{S}\left|\varphi_{\delta}\right|_{H^{1 / 2}\left(\Gamma^{s}\right)}^{2} \leq \gamma^{2}\left\|v_{h}\right\|^{2} .
\end{gathered}
$$

We point out that $\|\cdot\|_{\Phi}$ is indeed a norm on $\Phi$, since the elements of $\Phi$ vanish on $\partial \Omega$ (see Bertoluzza [2003]). One of the consequences of (26) is that the seminorm $\|\cdot\|$ is indeed a norm. In fact, given $v_{h} \in \mathcal{S}_{h}$ and letting $\varphi \in \Phi_{\delta}$ given by (26), provided (22) holds, it can be shown (see Bertoluzza [2003]) that

$$
\left\|v_{h}\right\|_{0, \Omega} \leq C\left(\left\|v_{h}\right\|+\|\varphi\|_{\Phi}\right) \leq C\left\|v_{h}\right\| .
$$

We shall discuss in the following sections whether and when this assumption is satisfied. We anticipate however that this will be another easy condition, that could be roughly summarized by: on each face $f$ of each $\partial \Omega^{s}$ the mesh $\mathcal{T}_{\delta}^{\Gamma^{s}}$ (induced by $\mathcal{T}_{\delta}^{\Sigma}$ ) is coarser than the two meshes $\mathcal{T}_{h}^{\Gamma^{s}}$ (induced by the two $\mathcal{T}_{h}^{s}$ relative to the sub-domains having $f$ in common).

\section{Basic Error Estimates}

We are now ready to analyze the problem (11) and derive abstract error estimates for it.

We start by looking in more detail to the operator $\mathcal{G}^{s}$. Thanks to the classical theory of mixed finite element (see Brezzi and Fortin [1991b]) we can prove the following Lemma.

Lemma 1. Assume that the inf-sup condition (24) is satisfied, and let $\varphi \in$ $L^{2}(\Sigma)$; then for every $s=1, \ldots, S$

$$
\left\|\mathcal{G}^{s}(\varphi)\right\|_{h, \frac{1}{2}, \Gamma^{s}} \leq C\|\varphi\|_{h, \frac{1}{2}, \Gamma^{s}}
$$

We point out that the norm $\|\cdot\|_{h, \frac{1}{2}, \Gamma^{s}}$, induced by the bilinear form $(u, v) \rightarrow \sum_{T \in \mathcal{T}_{h}^{\Gamma^{s}}} \int_{T} h_{T}^{-1} u v \mathrm{~d} x$ plays the role of a discrete $H^{1 / 2}\left(\Gamma^{s}\right)$ norm. Indeed we have the following lemma. 
Lemma 2. The following inverse inequality holds: for all $\theta_{h}^{s} \in \Theta_{h}^{s}$

$$
\left\|\theta_{h}^{s}\right\|_{H^{1 / 2}\left(\Gamma^{s}\right)} \leq C\left\|\theta_{h}^{s}\right\|_{h, \frac{1}{2}, \Gamma^{s}}
$$

Proof. We shall actually prove that (29) holds for all $\theta_{h}^{s} \in \mathcal{L}_{\kappa}^{1}\left(\mathcal{T}_{h}^{\Gamma^{s}}\right)$. It is well known that a function in $\mathcal{L}_{\kappa}^{1}\left(\mathcal{T}_{h}^{s}\right)$ is uniquely identified by its values at a set $\left\{x_{i}\right\}_{i}$ of nodes corresponding to the canonical Lagrange basis. Let $\theta_{h}^{s} \in \mathcal{L}_{\kappa}^{1}\left(\mathcal{T}_{h}^{\Gamma^{s}}\right)$ and let $w_{h} \in \mathcal{L}_{\kappa}^{1}\left(\mathcal{T}_{h}^{s}\right)$ be its finite element lifting, i.e., the function verifying $w_{h}^{s}\left(x_{i}\right)=\theta_{h}^{s}\left(x_{i}\right)$ at all nodes on $\Gamma^{s}$ and $w_{h}^{s}\left(x_{i}\right)=0$ at all other nodes. Clearly, $\left\|\theta_{h}^{s}\right\|_{H^{1 / 2}\left(\Gamma^{s}\right)} \leq C\left\|w_{h}^{s}\right\|_{H^{1}\left(\Omega^{s}\right)}$. Let us then bound the $H^{1}\left(\Omega^{s}\right)$ norm of $w_{h}^{s}$. By definition $w_{h}^{s}$ is different from 0 only on those tetrahedra $T \in \mathcal{T}_{h}^{s}$ which are adjacent to the boundary. Let $K$ be one of such tetrahedra and let $T_{i} \in \mathcal{T}_{h}^{\Gamma^{s}}, i=1, \ldots, m$ be the triangles that share one or more nodes with $K$. Thanks to usual arguments, we can write:

$$
\left\|w_{h}^{s}\right\|_{H^{1}(K)}^{2} \leq C h_{K}^{-1}\left\|w_{h}^{s}\right\|_{L^{2}(\partial K)}^{2} \leq C \sum_{i=1}^{m} h_{T_{i}}^{-1}\left\|w_{h}^{s}\right\|_{L^{2}\left(T_{i}\right)}^{2} .
$$

Adding with respect to all elements $K$ adjacent to $\Gamma^{s}$, we obtain that

$$
\left\|w_{h}^{s}\right\|_{H^{1}\left(\Omega^{s}\right)} \leq C\left\|\theta_{h}^{s}\right\|_{h, \frac{1}{2}, \Gamma^{s}},
$$

which implies (29).

Remark 1. Note that if we had assumed the quasi-uniformity of the triangulation $\mathcal{T}_{h}^{\Gamma^{s}}$, then (29) could easily be obtained by space interpolation, using the standard inverse inequality between the $H^{1}$ and the $L^{2}$ norms. This is however not the case, and in the above proof we only made use of the regularity of the mesh.

Lemma 2 trivially implies the continuity of $\mathcal{G}^{s}$ from $L^{2}\left(\Gamma^{s}\right)$ (endowed with the norm $\left.\|\cdot\|_{h, \frac{1}{2}, \Gamma^{s}}\right)$, to $H^{1 / 2}\left(\Gamma^{s}\right)$. However a stronger result holds, stated in the following theorem

Theorem 1. $\mathcal{G}^{s}(\cdot)$ is continuous from $H^{1 / 2}\left(\Gamma^{s}\right)$ to $H^{1 / 2}\left(\Gamma^{s}\right)$ :

$$
\left\|\mathcal{G}^{s}(\varphi)\right\|_{H^{1 / 2}\left(\Gamma^{s}\right)} \leq C\|\varphi\|_{H^{1 / 2}\left(\Gamma^{s}\right)} .
$$

Proof. First, we introduce the Clément interpolant $\theta_{I}^{s} \in \Theta_{h}^{s}$ of $\theta^{s}=\left.\varphi\right|_{\Gamma^{s}}$, which gives (see Clément [1975])

$$
\begin{aligned}
\left\|\theta_{I}^{s}\right\|_{H^{1 / 2}\left(\Gamma^{s}\right)} & \leq C\left\|\theta^{s}\right\|_{H^{1 / 2}\left(\Gamma^{s}\right)} \\
\left\|\theta^{s}-\theta_{I}^{s}\right\|_{h, \frac{1}{2}, \Gamma^{s}} & \leq C\left\|\theta^{s}\right\|_{H^{1 / 2}\left(\Gamma^{s}\right)} .
\end{aligned}
$$

Since $\mathcal{G}^{s}(\cdot)$ is linear and using the triangle inequality, we have

$$
\left\|\mathcal{G}^{s}\left(\theta^{s}\right)\right\|_{H^{1 / 2}\left(\Gamma^{s}\right)} \leq\left\|\mathcal{G}^{s}\left(\theta^{s}-\theta_{I}^{s}\right)\right\|_{H^{1 / 2}\left(\Gamma^{s}\right)}+\left\|\mathcal{G}^{s}\left(\theta_{I}^{s}\right)\right\|_{H^{1 / 2}\left(\Gamma^{s}\right)}=I+I I .
$$


Making use of Lemma 2, Lemma 1 and (31), we get

$$
\begin{aligned}
I=\left\|\mathcal{G}^{s}\left(\theta-\theta_{I}^{s}\right)\right\|_{H^{1 / 2}\left(\Gamma^{s}\right)} & \leq C\left\|\mathcal{G}^{s}\left(\theta-\theta_{I}^{s}\right)\right\|_{h, \frac{1}{2}, \Gamma^{s}} \\
& \leq C\left\|\theta-\theta_{I}^{s}\right\|_{h, \frac{1}{2}, \Gamma^{s}} \\
& \leq C\|\theta\|_{H^{1 / 2}\left(\Gamma^{s}\right)} .
\end{aligned}
$$

Moreover, since $\mathcal{G}^{s}\left(\theta_{I}^{s}\right)=\theta_{I}^{s}$ and using (31), we have

$$
I I=\left\|\mathcal{G}^{s}\left(\theta_{I}^{s}\right)\right\|_{H^{1 / 2}\left(\Gamma^{s}\right)} \leq C\|\theta\|_{H^{1 / 2}\left(\Gamma^{s}\right)},
$$

giving (30).

We can now prove our error estimate. From the definition (10) and assumption (27) we easily get that problem (11) has a unique solution. Let now $\psi_{I}$ be an interpolant of the exact solution $u$ in $\Phi_{\delta}$. For every $\Omega^{s}(s=1, \ldots, S)$ let $u_{I}^{s} \in V_{h}^{s}$ be defined as the unique solution of

$$
\left\{\begin{array}{l}
u_{I}^{s}=\mathcal{G}^{s}\left(\psi_{I}\right) \text { on } \Gamma^{s} \\
a_{s}\left(u_{I}^{s}, v_{h}^{s}\right)=\int_{\Omega^{s}} g v_{h}^{s} \mathrm{~d} x \quad \forall v_{h}^{s} \in V_{h}^{s} \cap H_{0}^{1}\left(\Omega^{s}\right) .
\end{array}\right.
$$

It is obvious that (32) has a unique solution. Let $u_{I}$ be equal to $u_{I}^{s}$ in each $\Omega^{s}$ $(s=1, \ldots, S)$. It is clear that $u_{I} \in \mathcal{S}_{h}$. We now set $e_{h}:=u_{I}-u_{h} \in \mathcal{S}_{h}$. Using the the definition (10) and adding and subtracting $u$ we have:

$$
\left\|e_{h}\right\|^{2}=a\left(e_{h}, e_{h}\right)=a\left(u_{I}-u, e_{h}\right)+a\left(u-u_{h}, e_{h}\right)=: I+I I .
$$

Using (11) and integrating $a\left(u, e_{h}\right)$ by parts in each $\Omega^{s}$ we obtain

$$
\begin{aligned}
I I=a\left(u-u_{h}, e_{h}\right) & =-\sum_{s=1}^{S} \int_{\Omega^{s}} g e_{h}^{s} \mathrm{~d} x+\sum_{s=1}^{S} \int_{\Gamma^{s}} \frac{\partial u}{\partial \mathbf{n}^{s}} e_{h}^{s} \mathrm{~d} x-\sum_{s=1}^{S} \int_{\Omega^{s}} g e_{h}^{s} \mathrm{~d} x \\
& =\sum_{s=1}^{S} \int_{\Gamma^{s}} \frac{\partial u}{\partial \mathbf{n}^{s}} e_{h}^{s} \mathrm{~d} x .
\end{aligned}
$$

As $e_{h} \in \mathcal{S}_{h}$, and using assumption (26) there will be a mother $\eta_{\delta} \in \Phi_{\delta}$ with $\left\|\eta_{\delta}\right\|_{\Phi} \leq C\left\|e_{h}\right\|$, such that $\mathcal{G}\left(\eta_{\delta}\right)=\left.e_{h}\right|_{\Sigma}$. Hence the continuity of $\partial u / \partial \mathbf{n}$, and the fact that $\eta_{\delta}$ is single-valued on the skeleton $\Sigma$ yield

$$
I I=\sum_{s=1}^{S} \int_{\Gamma^{s}} \frac{\partial u}{\partial \mathbf{n}^{s}}\left(e_{h}^{s}-\eta_{\delta}\right) \mathrm{d} x=\sum_{s=1}^{S} \int_{\Gamma^{s}} \frac{\partial u}{\partial \mathbf{n}^{s}}\left(\mathcal{G}^{s}\left(\eta_{\delta}\right)-\eta_{\delta}\right) \mathrm{d} x .
$$

We can now use the definition of $\mathcal{G}^{s}$ (see (15)) and subtract from $\partial u / \partial \mathbf{n}$ its best approximation $\mu_{I}^{s}$, thus obtaining

$$
I I=\sum_{s=1}^{S} \int_{\Gamma^{s}}\left(\frac{\partial u}{\partial \mathbf{n}^{s}}-\mu_{I}^{s}\right)\left(\mathcal{G}^{s}\left(\eta_{\delta}\right)-\eta_{\delta}\right) \mathrm{d} x .
$$


We remember now that $\mathcal{G}^{s}\left(\eta_{\delta}\right)=e_{h}^{s}$ on $\Gamma^{s}$ for all $s$. We also point out that (thanks to (22)) we can assume that the mean value of $\partial u / \partial \mathbf{n}^{s}-\mu_{I}^{s}$ on each $\Gamma^{s}$ is zero, so that we can use the $H^{1 / 2}$-seminorm of $e^{s}$ and $\eta_{\delta}$ instead of the norm in the estimate. Then we use Cauchy-Schwarz inequality, we use (26) for $\eta_{\delta}$, and (27) standard trace inequality in each $\Omega^{s}$ for $e^{s}$ to obtain

$$
\begin{aligned}
I I \leq \sum_{s=1}^{S}\left\|\frac{\partial u}{\partial \mathbf{n}^{s}}-\mu_{I}^{s}\right\|_{H^{-1 / 2}\left(\Gamma^{s}\right)} & \left(\left|e^{s}\right|_{H^{1 / 2}\left(\Gamma^{s}\right)}+\left|\eta_{\delta}\right|_{H^{1 / 2}\left(\Gamma^{s}\right)}\right) \\
& \leq\left(\sum_{s=1}^{S}\left\|\frac{\partial u}{\partial \mathbf{n}^{s}}-\mu_{I}^{s}\right\|_{H^{-1 / 2}\left(\Gamma^{s}\right)}^{2}\right)^{1 / 2}\left\|e_{h}\right\| .
\end{aligned}
$$

It remains to estimate $I$. After the obvious

$$
I=a\left(u_{I}-u, e_{h}\right) \leq\left\|u_{I}-u\right\|\left\|e_{h}\right\|
$$

we have to estimate $\left\|u-u_{I}\right\|$. Using the definition (32) of $u_{I}^{s}$ we can apply the usual theory for estimating the error for each Dirichlet problem in $\Omega^{s}$. Thanks to (20) we have first

$$
\left\|u-u_{I}^{s}\right\|_{1, \Omega^{s}} \leq C\left(\inf _{v_{h}^{s} \in V_{h}^{s}}\left\|u-v_{h}^{s}\right\|_{1, \Omega^{s}}+\left\|u-u_{I}^{s}\right\|_{H^{1 / 2}\left(\Gamma^{s}\right)}\right) .
$$

It is then clear that the crucial step is to estimate $\left\|u-u_{I}^{s}\right\|_{H^{1 / 2}\left(\Gamma^{s}\right)}$, for each $s$.

To this aim let us introduce an interpolant $\chi_{I}^{s} \in \Theta_{h}^{s}$ of $\left.u\right|_{\Gamma^{s}}$. We can write

$$
\begin{aligned}
\left\|u-u_{I}^{s}\right\|_{H^{1 / 2}\left(\Gamma^{s}\right)} \equiv\left\|u-\mathcal{G}^{s}\left(\psi_{I}\right)\right\|_{H^{1 / 2}\left(\Gamma^{s}\right)} & \leq\left\|u-\chi_{I}^{s}\right\|_{H^{1 / 2}\left(\Gamma^{s}\right)}+\left\|\chi_{I}^{s}-\mathcal{G}^{s}(u)\right\|_{H^{1 / 2}\left(\Gamma^{s}\right)} \\
& +\left\|\mathcal{G}^{s}(u)-\mathcal{G}^{s}\left(\psi_{I}\right)\right\|_{H^{1 / 2}\left(\Gamma^{s}\right)}
\end{aligned}
$$

Since $\chi_{I}^{s}=\mathcal{G}^{s}\left(\chi_{I}^{s}\right)$ and using Theorem 1 , we easily get $\left\|\chi_{I}^{s}-\mathcal{G}^{s}(u)\right\|_{H^{1 / 2}\left(\Gamma^{s}\right)}=$ $\left\|\mathcal{G}^{s}\left(\chi_{I}^{s}-u\right)\right\|_{H^{1 / 2}\left(\Gamma^{s}\right)} \leq C\left\|u-\chi_{I}^{s}\right\|_{H^{1 / 2}\left(\Gamma^{s}\right)}$. By a similar argument we obtain $\left\|\mathcal{G}^{s}\left(u-\psi_{I}\right)\right\|_{H^{1 / 2}\left(\Gamma^{s}\right)} \leq\left\|u-\psi_{I}\right\|_{H^{1 / 2}\left(\Gamma^{s}\right)}$.

We can then collect (33)-(38) and (39)-(41) in the following theorem.

Theorem 2. Assume that the assumptions of Section 2 on the decomposition and on the discretizations are satisfied. Assume that the operators $\mathcal{G}^{s}$ are constructed as in (14)-(16). Let $u$ be the exact solution of (1) and $u_{h}$ be the solution of (11). Then we have

$$
\begin{array}{r}
\left\|u-u_{h}\right\|^{2} \leq C \sum_{s=1}^{S}\left(\inf _{v_{h}^{s} \in V_{h}^{s}}\left\|u-v_{h}^{s}\right\|_{1, \Omega^{s}}^{2}+\inf _{\mu_{h}^{s} \in M_{h}^{s}}\left\|\frac{\partial u}{\partial \mathbf{n}^{s}}-\mu_{h}^{s}\right\|_{H^{-1 / 2}\left(\Gamma^{s}\right)}^{2}\right) \\
+\inf _{\varphi_{\delta} \in \Phi_{\delta}}\left\|u-\varphi_{\delta}\right\|_{H^{1 / 2}(\Sigma)^{2}}^{2}
\end{array}
$$




\section{Examples and Remarks}

In this section we want to show an example of finite element discretizations that satisfy the abstract assumptions of Section 2, and derive the corresponding error bounds in terms of suitable powers of $h$.

We do not discuss the assumptions on the decomposition of $\Omega$ into the $\Omega^{s}$. We just remark once more that it does not need to be compatible: for instance, the intersection of the closures of two different $\Omega^{s}$ can be a face of one of them and only a piece of a face of the other.

We discuss instead the choice of the finite element spaces $\Phi_{\delta}, V_{h}^{s}$, and $M_{h}^{s}$.

Assume that we are given an integer number $k \geq 1$.

For every $T$ in the triangulation $\mathcal{T}_{\delta}^{\Sigma}$ of the skeleton $\Sigma$ we choose $\mathcal{P}_{T}:=$ $\mathbb{P}_{k}(T)$, the space of polynomials of degree $\leq k$ on $T$. The space $\Phi_{\delta}$, according to (17), becomes then

$$
\Phi_{\delta}:=\left\{\varphi \in \Phi \text { such that } \varphi_{\mid T} \in \mathbb{P}_{k}(T), \quad T \in \mathcal{T}_{\delta}^{\Sigma}\right\}=\mathcal{L}_{k}^{1}\left(\mathcal{T}_{\delta}^{\Sigma}\right) \cap \Phi
$$

(we recall that the elements of $\Phi$ have to vanish on $\partial \Omega$ so we need to take the intersection of $\mathcal{L}_{k}^{1}\left(\mathcal{T}_{\delta}^{\Sigma}\right)$ with $\Phi$ in order to properly define $\left.\Phi_{\delta}\right)$. For each $s$ and for every $T$ in the triangulation $\mathcal{T}_{h}^{\Gamma^{s}}$ of $\Gamma^{s}$ we take instead as $\mathcal{Q}_{T}$ the space $\mathcal{Q}_{T}:=\mathbb{P}_{k-1}(T)$. According to $(21)$ the space $M_{h}^{s}$ becomes then

$$
M_{h}^{s}:=\left\{\mu \in L^{2}\left(\Gamma^{s}\right): \mu_{\mid T} \in \mathbb{P}_{k-1}(T), \quad T \in \mathcal{T}_{h}^{\Gamma^{s}}\right\}=\mathcal{L}_{k}^{0}\left(\mathcal{T}_{h}^{\Gamma^{s}}\right) .
$$

We point out that $\Phi_{\delta}$ is made of continuous functions, while $M_{h}^{s}$ is made of functions that are, a priori, totally discontinuous from one element to another.

The choice of each $V_{h}^{s}$ will be slightly more elaborate. For each tetrahedron $K \in \mathcal{T}_{h}^{s}$ with no faces belonging to $\Gamma^{s}$ we take $\mathcal{P}_{K}:=\mathbb{P}_{k}$. If instead $K$ has a face $f$ on $\Gamma^{s}$ we consider the cubic function $b_{f}$ on $K$ that vanishes on the three remaining internal faces of $K$, and we augment the space $\mathbb{P}_{k}$ with the space $B_{k+2}^{f}$ obtained multiplying $b_{f}$ times the functions in $\mathcal{Q}_{f} \equiv \mathbb{P}_{k-1}(f)$ (that is the space of polynomials of degree $\leq k-1$ on $f$ : remember that the face $f$ will be one of the triangles $\left.T \in \mathcal{T}_{h}^{\Gamma^{s}}\right)$. If $K$ has another face on $\Gamma^{s}$ we repeat the operation, augmenting further the space $\mathbb{P}_{k}$. In summary

$$
\mathcal{P}_{K}:=\mathbb{P}_{k}(K)+\left\{\bigoplus_{f \subset \Gamma^{s}} B_{k+2}^{f}\right\} \equiv \mathbb{P}_{k}+\left\{\bigoplus_{f \subset \Gamma^{s}} b_{f} \mathbb{P}_{k-1}(f)\right\} .
$$

We note that $\bigoplus b_{f} \mathbb{P}_{k-1}(f)$ is a direct sum, but its sum with $\mathbb{P}_{k}(K)$ is not direct whenever $k \geq 3$. This however will not be a problem for the following developments.

We can now discuss the various abstract assumptions that have been made in Section 2. To start with, condition (22) is obviously satisfied. Similarly, (20) holds as shown for instance in Bernardi et al. [to appear]. We consider then the inf-sup condition (24).

Lemma 3. Let $M_{h}^{s}$ and $\Theta_{h}^{s}$ be constructed as in (44) and in (19) with (45), respectively. Then the inf-sup condition (24) holds true. 
Proof. For every $\mu_{h}^{s} \in M_{h}^{s}$ we construct $v_{h}^{s} \in V_{h}^{s}$ as

$$
v_{h}^{s}=\sum_{T \in \mathcal{T}_{h}^{\Gamma^{s}}} h_{T} b_{T} \mu_{h}^{s}
$$

where as before $b_{T}$ is the cubic function on $K$ (the tetrahedron having $T$ as one of its faces) vanishing on the other three faces of $K$ and having mean value 1 on $T$. It is not too difficult to check that

$$
\left\|\mu_{h}^{s}\right\|_{h,-\frac{1}{2}, \Gamma^{s}}\left\|v_{h}^{s}\right\|_{h, \frac{1}{2}, \Gamma^{s}} \leq C \int_{\mathcal{T}_{h}^{\Gamma^{s}}} v_{h}^{s} \mu_{h}^{s}
$$

that is precisely the inf-sup condition (24) that we need.

We consider now the other inf-sup that is involved in the present scheme (although we did not write it as an inf-sup), that is the bound on the mother (26). By applying the technique of Babuska [1973] it is not difficult to realize that if $\mathcal{T}_{\delta}^{\Sigma}$ is "coarse enough" on each face, compared with the meshes of the two sub-domains having that face in common, then

$$
\inf _{\varphi_{\delta} \in \mathcal{L}_{k}^{1}\left(\mathcal{T}_{\delta}^{T^{s}}\right) \backslash\{0\}} \sup _{\mu_{h}^{s} \in M_{h}^{s} \backslash\{0\}} \frac{\int_{\Gamma^{s}} \varphi_{\delta} \mu_{h}^{s} \mathrm{~d} x}{\left\|\mu_{h}^{s}\right\|_{H^{-1 / 2}\left(\Gamma^{s}\right)}\left\|\varphi_{\delta}\right\|_{H^{1 / 2}\left(\Gamma^{s}\right)}}>\gamma_{0} .
$$

It is now easy to see that (48) implies (26): let $v_{h}^{s} \in \mathcal{S}_{h}$, then, by definition, there exists $\varphi_{\delta} \in \Phi_{\delta}$ such that $\left.v_{h}^{s}\right|_{\Sigma}=\varphi_{\delta}$. Letting $\check{\varphi}^{s}=\left(1 /\left|\Gamma^{s}\right|\right) \int_{\Gamma^{s}} \varphi_{\delta} \mathrm{d} x$ we have that $\varphi_{\delta}-\check{\varphi}^{s} \in \mathcal{L}_{k}^{1}\left(\mathcal{T}_{\delta}^{\Gamma^{s}}\right)$. Let now $\mu_{h}^{*} \in M_{h}^{s}$ be the element that realizes the supremum in (48) for such an element of $\mathcal{L}_{k}^{1}\left(\mathcal{T}_{\delta}^{\Gamma^{s}}\right)$. Using (48), and then (14), we obtain

$$
\begin{aligned}
\gamma_{0}\left\|\mu_{h}^{*}\right\|_{H^{-1 / 2}\left(\Gamma^{s}\right)}\left\|\varphi_{\delta}-\check{\varphi}^{s}\right\|_{H^{1 / 2}\left(\Gamma^{s}\right)} & \leq \int_{\Gamma^{s}} \mu_{h}^{*}\left(\varphi_{\delta}-\check{\varphi}^{s}\right) \mathrm{d} x \\
& =\int_{\Gamma^{s}} \mu_{h}^{*} \mathcal{G}^{s}\left(\varphi_{\delta}-\check{\varphi}^{s}\right) \mathrm{d} x .
\end{aligned}
$$

Now, since $\varphi_{\delta}-\check{\varphi}^{s}$ has zero mean value on $\Gamma^{s}$, the same is true for $\mathcal{G}^{s}\left(\varphi_{\delta}-\check{\varphi}^{s}\right)$ (see (14) and (22)). Then, denoting by $\check{v}^{s}=\left(1 /\left|\Gamma^{s}\right|\right) \int_{\Gamma^{s}} v_{h}^{s} \mathrm{~d} x$ the average of $v_{h}^{s}$ on $\Gamma^{s}$, we have

$$
\begin{aligned}
\int_{\Gamma^{s}} \mu_{h}^{*} \mathcal{G}^{s}\left(\varphi_{\delta}-\check{\varphi}^{s}\right) \mathrm{d} x & =\int_{\Gamma^{s}} \mu_{h}^{*}\left(v_{h}^{s}-\check{v}^{s}\right) \mathrm{d} x \\
& \leq\left\|\mu_{h}^{*}\right\|_{H^{-1 / 2}\left(\Gamma^{s}\right)}\left|v_{h}^{s}\right|_{H^{1 / 2}\left(\Gamma^{s}\right)} \\
& \leq\left\|\mu_{h}^{*}\right\|_{H^{-1 / 2}\left(\Gamma^{s}\right)}\left|v_{h}^{s}\right|_{1, \Omega^{s}}
\end{aligned}
$$

that, since $\left|\varphi_{\delta}\right|_{H^{1 / 2}\left(\Gamma^{s}\right)}=\left|\varphi_{\delta}-\breve{\varphi}^{s}\right|_{H^{1 / 2}\left(\Gamma^{s}\right)} \simeq\left\|\varphi_{\delta}-\breve{\varphi}^{s}\right\|_{H^{1 / 2}\left(\Gamma^{s}\right)}$, joined with (49) immediately implies (26).

We can collect the previous results, together with the abstract error estimates of the previous section, in the following theorem. 
Theorem 3. Assume that the assumptions on the decompositions $\mathcal{T}_{\delta}^{\Sigma}$ and $\mathcal{T}_{h}^{s}$ of Section 2 are satisfied, and assume that the spaces $\Phi_{\delta}, M_{h}^{s}$ and $V_{h}^{s}$ are defined as in (43), (44) and (18) with (45), respectively. Assume finally that (48) holds. Then we have

$$
\left\|u_{h}-u\right\| \leq C\left(|h|^{k}+|\delta|^{k}\right)\|u\|_{k+1, \Omega}
$$

The proof follows immediately from Theorem 1, the results of this section, and usual approximation estimates.

We end this section with some observations on the actual implementation of the method when the bubble stabilization (45) is used.

Indeed, let us see how the computation of the generation operators $\mathcal{G}^{s}$ can be performed in practice. Assume that we are given a function $\varphi$ in, say, $L^{2}\left(\Gamma^{s}\right)$. We recall that, to compute $\widetilde{\theta_{h}^{s}}=\mathcal{G}^{s}(\varphi)$, we have to find the pair $\left(\widetilde{\theta_{h}^{s}}, \widetilde{\mu_{h}^{s}}\right) \in \Theta_{h}^{s} \times M_{h}^{s}$ such that

$$
\begin{gathered}
\int_{\Gamma^{s}}\left(\varphi-\widetilde{\theta_{h}^{s}}\right) \mu_{h}^{s} \mathrm{~d} x=0 \quad \forall \mu_{h}^{s} \in M_{h}^{s}, \\
\sum_{T \in \mathcal{T}_{h}^{\Gamma^{s}}} \int_{T} h_{T}^{-1}\left(\varphi-\widetilde{\theta_{h}^{s}}\right) \theta_{h}^{s} \mathrm{~d} x+\int_{\Gamma^{s}} \widetilde{\mu_{h}^{s}} \theta_{h}^{s} \mathrm{~d} x=0 \quad \forall \theta_{h}^{s} \in \Theta_{h}^{s} .
\end{gathered}
$$

We also recall that, with the choice (45), the space $\Theta_{h}^{s}$ can be written as $\Theta_{h}^{s}=$ $\mathcal{L}_{k}^{1}\left(\mathcal{T}_{h}^{\Gamma^{s}}\right)+B_{k+2}\left(\mathcal{T}_{h}^{\Gamma^{s}}\right)$ where $\mathcal{L}_{k}^{1}\left(\mathcal{T}_{h}^{\Gamma^{s}}\right)$ is, as before, the space of continuous piecewise polynomials of degree $k$ on the mesh $\mathcal{T}_{h}^{\Gamma^{s}}$, and $B_{k+2}\left(\mathcal{T}_{h}^{\Gamma^{s}}\right)$ is the space of bubbles of degree $k+2$, always on $\mathcal{T}_{h}^{\Gamma^{s}}$. In order to write is as a direct sum we introduce the space

$$
W^{s}=\left\{\theta_{h}^{s} \in \Theta_{h}^{s} \text { such that } \int_{\Gamma^{s}} \theta_{h}^{s} \mu_{h}^{s} \mathrm{~d} x=0 \forall \mu_{h}^{s} \in M_{h}^{s}\right\}
$$

We can then split in a unique way $\widetilde{\theta_{h}^{s}}=\widetilde{w}+\widetilde{b}$ with $\widetilde{w} \in W^{s}$ and $\widetilde{b}$ in $B_{k+2}\left(\mathcal{T}_{h}^{\Gamma^{s}}\right)$. It is now clear that $\widetilde{b}$ can be computed immediately from (52) that becomes:

$$
\int_{\Gamma^{s}}(\varphi-\widetilde{b}) \mu_{h}^{s} \mathrm{~d} x=0 \quad \forall \mu_{h}^{s} \in M_{h}^{s} .
$$

Once $\widetilde{b}$ is known, one can compute $\widetilde{w}$ from (53) that easily implies

$$
\sum_{T \in \mathcal{T}_{h}^{\Gamma^{s}}} \int_{T} h_{T}^{-1}(\varphi-\widetilde{w}) w, \mathrm{~d} x=\sum_{T \in \mathcal{T}_{h}^{\Gamma^{s}}} \int_{T} h_{T}^{-1} \widetilde{b} w, \mathrm{~d} x \quad \forall w \in W^{s} .
$$

In this way the saddle point problem (52)-(53) splits into two smaller subproblems, each with a symmetric and positive definite matrix. In particular (55) can be solved element by element, so that (56) is the only true system to be solved. 


\section{Relaxing the continuity of the Mothers}

One of the main advantages of the present method (and in general of all non conforming domain decomposition methods) is the freedom given by the possibility of meshing and treating each sub-domain independently of the others. In our approach however, the discretization $\Phi_{\delta}$ of $H^{1 / 2}(\Sigma)$ is required to be continuous. Such request can be relaxed by defining $\Phi_{\delta}$ face by face and asking for continuity within each face but allowing the elements of $\Phi_{\delta}$ to jump across the boundary between two adjacent faces. More precisely, considering a splitting of the skeleton $\Sigma$ in disjoint faces $\Sigma=\cup f$ (with $f=\Gamma^{s} \cap \Gamma^{\ell}$ for some $s, \ell=1, \ldots, S)$ we can introduce for each face a family of triangulations $\mathcal{T}_{\delta}^{f}$ and consider a corresponding space $\Phi_{\delta}^{f} \subset H^{1 / 2}(f)$ of piecewise polynomials. The global space $\Phi_{\delta}$ could then be defined by

$$
\Phi_{\delta}=\left\{\varphi_{\delta} \in L^{2}(\Sigma) \text { with }\left.\varphi_{\delta}\right|_{f} \in \Phi_{\delta}^{f} \text { for all faces } f \text { of } \Sigma\right\} .
$$

Such a choice has several advantages, in particular from the point of view of implementation. Each face can be meshed independently of the other faces. Moreover, each node on $\Sigma$ belongs to only one face $f$ and therefore it only "sees" two sub-domains. This greatly simplifies the data structure needed for describing the elements of $\Phi_{\delta}$ and the manipulations of such elements and of their interaction with other elements.

The analysis presented in the previous section needs then to be modified in order to take the discontinuity of the mothers into account. In particular, if the elements of $\Phi_{\delta}$ are discontinuous, the space $\Phi_{\delta}$ is not a subspace of $H^{1 / 2}(\Sigma)$, and therefore bounds like $\left\|\mathcal{G}^{s}\left(u-\psi_{I}\right)\right\|_{H^{1 / 2}\left(\Gamma^{s}\right)} \leq\left\|u-\psi_{I}\right\|_{H^{1 / 2}\left(\Gamma^{s}\right)}$ would not make sense. A completely revised analysis is carried out in a further work in preparation, and results in an almost optimal estimate (with the loss of a logarithmic factor). We just point out that the analysis of Section 3 could still be applied if the space $\Phi_{\delta}^{c}=\Phi_{\delta} \cap H^{1 / 2}(\Sigma)$ has good approximation properties. Such space is the one where one should choose the best approximation $\psi_{I}$. This is indeed a very special case: in general such space does not provide a good approximation. It may very well happen that it contains only the function $\varphi_{\delta}=0$. A case in which the space $\Phi_{\delta}^{c}$ does provide good approximation is the case in which the meshes on two adjacent faces share a sufficiently fine set of common nodes (in particular the case when, restricted to the common edge, the nodes of the two (or more) meshes are one a subset of the other). Though this is quite an heavy restriction to the freedom given by the possibility of using discontinuous mothers, such a case would still have many advantages from the implementation point of view, while retaining the optimal error estimate. Remark that the subspace $\Phi_{\delta}^{c}$ would only be used for analyzing the method, while its implementation fully relies on the discontinuous space $\Phi_{\delta}$. 


\section{References}

I. Babuska. The finite element method with lagrangian multipliers. Numer. Math., 20:179-192, 1973.

C. Baiocchi, F. Brezzi, and L. D. Marini. Stabilization of galerkin methods and applications to domain decomposition. In A. Bensoussan et al., editor, Future Tendencies in Computer Science, Control and Applied Mathematics, volume 653 of Lecture Notes in Computer Science, pages 345-355. SpringerVerlag, 1992.

F. B. Belgacem and Y. Maday. The mortar element method for three dimensional finite elements. RAIRO Mathematical Modelling and Numerical Analysis, 31(2):289-302, 1997.

C. Bernardi, Y. Maday, and A. T. Patera. Domain decomposition by the mortar element method. In H. K. ans M. Garbey, editor, Asymptotic and Numerical Methods for Partial Differential Equations with Critical Parameters, pages 269-286. N.A.T.O. ASI, Kluwer Academic Publishers, 1993.

C. Bernardi, Y. Maday, and F. Rapetti. Discrétisations variationnelles de problèmes aux limites elliptiques. Mathématiques et Applications. SMAI, to appear.

S. Bertoluzza. Analysis of a stabilized three-fields domain decomposition method. Numer. Math., 93(4):611-634, 2003. ISSN 0029-599X.

F. Brezzi and M. Fortin. Mixed and Hybrid Finite Element Methods. SpringerVerlag, New-York, 1991a.

F. Brezzi and M. Fortin. Mixed and hybrid finite element methods. SpringerVerlag, New York, 1991b. ISBN 0-387-97582-9.

F. Brezzi, L. P. Franca, L. D. Marini, and A. Russo. Stabilization techniques for domain decomposition methods with nonmatching grids. In Proc. from the IX International Conference on Domain Decomposition Methods, June 1996, Bergen, Norway, 1997.

F. Brezzi and L. D. Marini. A three field domain decomposition method. Contemp. Math., 157:27-34, 1994.

F. Brezzi and L. D. Marini. Error estimates for the three-field formulation with bubble stabilization. Math. of Comp., 70:911-934, 2000.

A. Buffa. Error estimates for a stabilized domain decomposition method with nonmatching grids. Numer. Math., 90(4):617-640, 2002.

P. Clément. Approximation by finite element functions using local regularization. RAIRO Anal. Numér., 9:77-84, 1975.

R. Hoppe, Y. Iliash, Y. Kuznetsov, Y. Vassilevski, and B. Wohlmuth. Analysis and parallel implementation of adaptive mortar element methods. East West J. Num. An., 6(3):223-248, 1998.

B. Wohlmuth. Discretization Methods and Iterative Solvers Based on Domain Decomposition, volume 17 of Lecture Notes in Computational Science and Engineering. Springer, 2001. 